\title{
Dos nuevos instrumentos de medida romanos hallados en Fresno Alhándiga (Salamanca)*
}

\section{Two new Roman measuring instruments from Fresno Alhándiga (Salamanca)}

Verónica Pérez de Dios

Investigadora independiente.

E-mail: veropdd@gmail.com

Orcid ID: https://orcid.org/0000-0002-1094-9944

\section{María de los Reyes de Soto García}

Instituto de Arqueología de Mérida

(CSIC-Junta de Extremadura).

E-mail: reyesdesoto@gmail.com

Orcid ID: https://orcid.org/0000-0002-3391-5616

Resumen: Las sociedades en las que se llevan a cabo intercambios de mercancías, como es el caso del mundo romano, desarrollan instrumentos de medida. En este artículo se presentan dos elementos de medida de cronología romana encontrados en un yacimiento rural de la provincia de Salamanca, Los Villares (Fresno Alhándiga). El hallazgo de este tipo de materiales en la provincia de Salamanca es significativamente escaso, no existen hasta el momento materiales comparables, de ahí la relevancia de los mismos. El lanx y el aequipodium de este trabajo estarían indicando unos contactos y la pervivencia de las vías de aprovisionamiento en la provincia de Salamanca por lo menos hasta la Tardoantigüedad.

Palabras Clave: aequipodium, medidas, pesas, poblamiento rural, Vía de la Plata.

CAUN 28 (2020): [1-10] 213-222

ISSN: 1133-1542. ISSN-e: 2387-1814

DOI: http://doi.org/10.15581/012.28.010
Abstract: Those societies where goods are exchanged, as it was the case of the Roman world, tend to develop measure devices. In this article, we introduce two measure elements from Roman chronology which were discovered in a rural site called, Los Villares (Fresno Alhándiga), in the province of Salamanca. The discovery of this type of material is highly relevant as it is remakably scarce in this province and there are no similar pieces up to now. Both the lanx and the aequipodium presented in this work point to quotidian market contacts and the survival of the provision routes in the province of Salamanca as far as the late-ancient times.

Keywords: aequipodium, measures, weight, rural settlement, Via de la Plata.

\footnotetext{
* Agradecer a la Junta de Castilla y León y especialmente a Esther González, Arqueóloga Territorial de Salamanca, su ayuda y financiación económica para el estudio y campañas arqueológicas desarrolladas en el enclave.
} 


\section{INTRODUCCIÓN}

A necesidad de cuantificar cantidades está presente en la sociedad romana, de ahí que los instrumentos de medida sean más o menos habituales ${ }^{1}$. Una revisión de materiales procedentes de excavaciones o de colecciones de antigüedades depararía, con casi total seguridad, la presencia de pesos o contrapesos, incluso de balanzas o romanas. Sin embargo, la publicación de los mismos suele quedar relegada a catálogos de piezas o, en el mejor de los casos, a una publicación propia si posee una decoración peculiar o algún tipo de inscripción.

Los instrumentos de medida son elementos básicos en las sociedades en las que existen intercambios de mercancías ya que era necesario medir de alguna manera en los intercambios de bienes. El origen de las balanzas se puede remontar hasta esos momentos en los que se establece una relación entre la necesidad de medir cantidades exactas y los procesos de mercantilización (Pares 2013: 508, 522-524). Las representaciones más antiguas conservadas se retrotraen al antiguo Egipto, y se trata únicamente de un brazo con dos platillos. Con el tiempo el sistema se fue refinando, pero el método siguió siendo el mismo: la compensación de un elemento a pesar con un contrapeso de referencia.

En este artículo se presentan dos piezas encontradas en el yacimiento de Los Villares (Fresno Alhándiga, Salamanca) que formarían parte de elementos de medida: un lanx y un aequipondium, ambos realizados en bronce, y hallados durante las labores de excavación que se están desarrollando en dicho enclave desde el año 2015.

\section{LOS INSTRUMENTOS DE PESAR EN EL MUNDO ROMANO}

En el mundo romano existieron distintos instrumentos de medida, el mismo Estado romano se interesó por la difusión de pesos estandarizados que facilitaran los intercambios por todo el Imperio (Pérez Zurita, 2011: 143), e incluso existía un control de fiabilidad como el documentado en una statera de Valencia, entre otras (Aranegui, 1989; Aranegui, 1991). El instrumento más sencillo fue la balanza o librae con brazos de la misma longitud y dos platillos, empleada para medir elementos de menor tamaño y fácilmente transportables. Se componen de una barra (scapus) de la que penden dos platillos (lances) unidos a la misma mediante una cadenita y su mecanismo se basa en colocar en un plato el elemento a medir y en el otro las pesas cuyo peso se conoce previamente, hasta conseguir la horizontalidad del scapus. Similar sería la balanza de brazos asimétricos, en la que uno de los brazos se alarga, gradúa y por lo tanto marca la medida gracias a un contrapeso. Un tercer tipo sería la staterae o «romana» con el dispositivo del que se suspende el instrumento descentrado, y del que resultan dos brazos diferentes. Las más frecuentes fueron las que tenían una

\footnotetext{
1 La mayoría de los autores que se han adentrado en este tema remarcan la poca presencia de estas piezas, pero no su total ausencia.
} 
barra (scapus) con varios dispositivos de suspensión que no estaban centrados ( fulcrum). El elemento a medir se suspendía de un gancho o mediante un platillo, y en el brazo opuesto se colocaba un contrapeso (aequipondium) (Erice Lacabe, 1986: 216; Chaves y Pliego, 2007; Cebrián y Hortelano, 2017: 202-204). Las balanzas necesitaban de unos pesos o pondera realizados de distintos materiales y formas, al igual que los contrapesos de las statera, también hechos de distintos materiales y con distintas formas, pudiendo ser desde simples bolas hasta aequipondia figurados.

Tanto librae, como staterae, pondus o aequipondia, elementos todos ellos necesarios para medir, son un tema poco tratado en la bibliografía arqueológica. Además, tradicionalmente son piezas que suelen recuperarse en colecciones de anticuarios, lo que limita la posibilidad de conocer su contexto e incluso su procedencia (Fernández Gómez, 1991; Corti, 2001: 196). En general, pocas son las publicaciones que hacen referencia a los elementos de medir contando que la primera sistematización de balanzas y ponderales de Hispania fue realizada en 2007 (Chaves y Pliego, 2007: 237250). No obstante, con anterioridad ya se habían ido publicando ejemplos, como las 16 balanzas y 18 pesos de Conimbriga (Ponte, 1979), algunos ejemplares de Andalucía (Chaves, 1982) o la exposición de Bronces en España donde se recopiló algunos de los aequipondia hallados (Caballero, 1990), a lo que se ha ido sumando una puesta al día realizada en 1994 (Pozo, 1994). Poco a poco el número de pesas y balanzas va aumentando, por lo que a los catálogos antes mencionados habría que añadir una pieza en Sofuentes (Erice Lacabe, 2014; Erice Lacabe, 2015) y las que se presenta en este artículo halladas en el yacimiento de Los Villares (Fresno Alhándiga, Salamanca). Pero, como señalaron R. Cebrián e I. Hortelano para poder encontrar más elementos de pesar se deben revisar memorias de excavación, lo que complica sustancialmente su estudio (Cebrián y Hortelano 2017: 205).

\section{LOS VILLARES (FRESNO ALHÁNDIGA, SALAMANCA)}

Los Villares es un yacimiento ubicado en el término municipal de Fresno Alhándiga (Salamanca) en terrenos de la fértil vega del río Alhándiga, uno de los afluentes más importantes del río Tormes (Figura 1). Hasta el año 2014 el enclave estaba únicamente reconocido como una concentración en superficie de materiales cerámicos y constructivos ubicado en la ribera de un pequeño regato. Sin embargo, durante las prospecciones realizadas enmarcadas en un proyecto de poblamiento romano del valle medio del río Tormes se descubrió un error en la ubicación del núcleo central en enclave, siendo la parte que estaba catalogada en el Inventario Arqueológico una parte residual.

Los trabajos de excavación comenzaron en el año 2015 tras el descubrimiento casual de un pavimento de opus tesselatum durante la realización de varios sondeos polínicos. En la primera campaña de excavación los esfuerzos se concentraron en sacar a la luz los restos del pavimento de mosaico, con el motivo de conocer su estado de conservación y extensión, a la vez que comprobar si existían más pavimentos de este tipo. Los trabajos arqueológicos depararon parte de las estructuras termales 


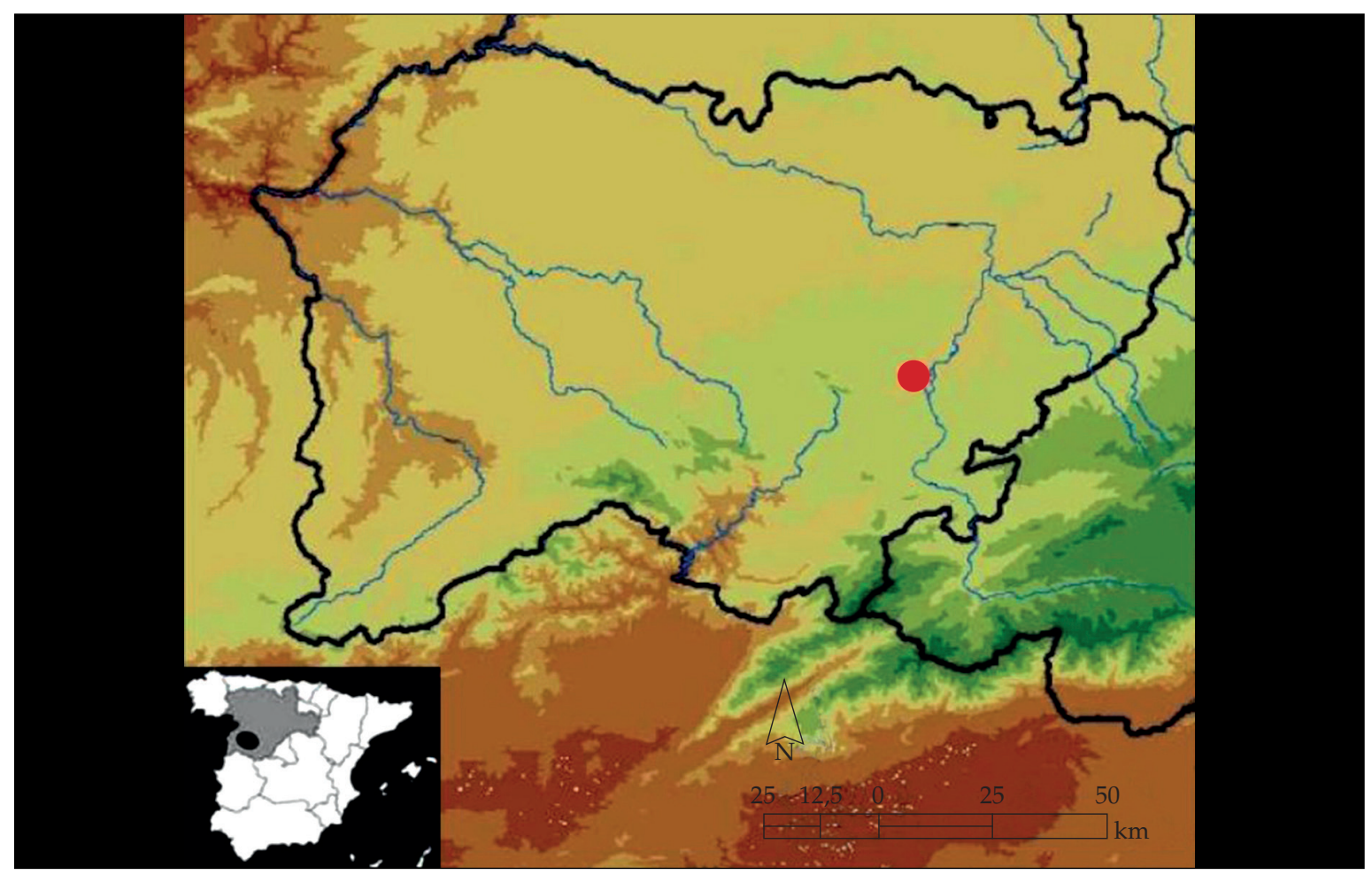

Figura 1

Mapa con la ubicación de Los Villares (Fresno Alhándiga, Salamanca)

de un asentamiento rural, habiéndose documentado varias estancias de las termas pavimentadas con mosaico y opus signinum y parte de su hipocaustum que conservaba las pilae en perfecto estado (Pérez y de Soto, 2017). Tras esta primera campaña se llevó a cabo un estudio geofísico para intentar dilucidar la tipología y límites de las estructuras con el fin de garantizar la conservación de todo el enclave. Con posterioridad, se han llevado a cabo otras dos campañas arqueológicas en los años 2017 y 2018 en los puntos más sensibles del enclave, marcados por los análisis no destructivos con el deseo de comprobar la fiabilidad de los datos y el estado de los restos, ya que las profundidades que se marcaban (unos $20-30 \mathrm{~cm}$ de profundidad) preocupaban por las labores agrícolas que se desarrollan en los campos (de Soto y Pérez, 2019). Las tres campañas de excavación han puesto al descubierto una pequeña parte de un asentamiento rural de grandes dimensiones en los que restos como cerámicas de importación, materiales metálicos, monedas, fragmentos de mosaico y pinturas murales son abundantes pero fragmentarios (de Soto y Pérez, 2019) (Figura 2).

El hallazgo de material metálico en Los Villares es más o menos habitual, tanto en excavación como en prospección. A lo largo de los años que el equipo lleva trabajando en el yacimiento se han podido recoger varias monedas tardías, clavos, hierros informes e incluso dos fragmentos de un colador de bronce bastante deformado del que solo ha quedado el vaso (Figura 3). Esta abundancia y variabilidad de materiales va en consonancia a otros materiales mucho más abundantes como son las cerámicas y el material constructivo, como tegulae, imbrex o tubuli. 


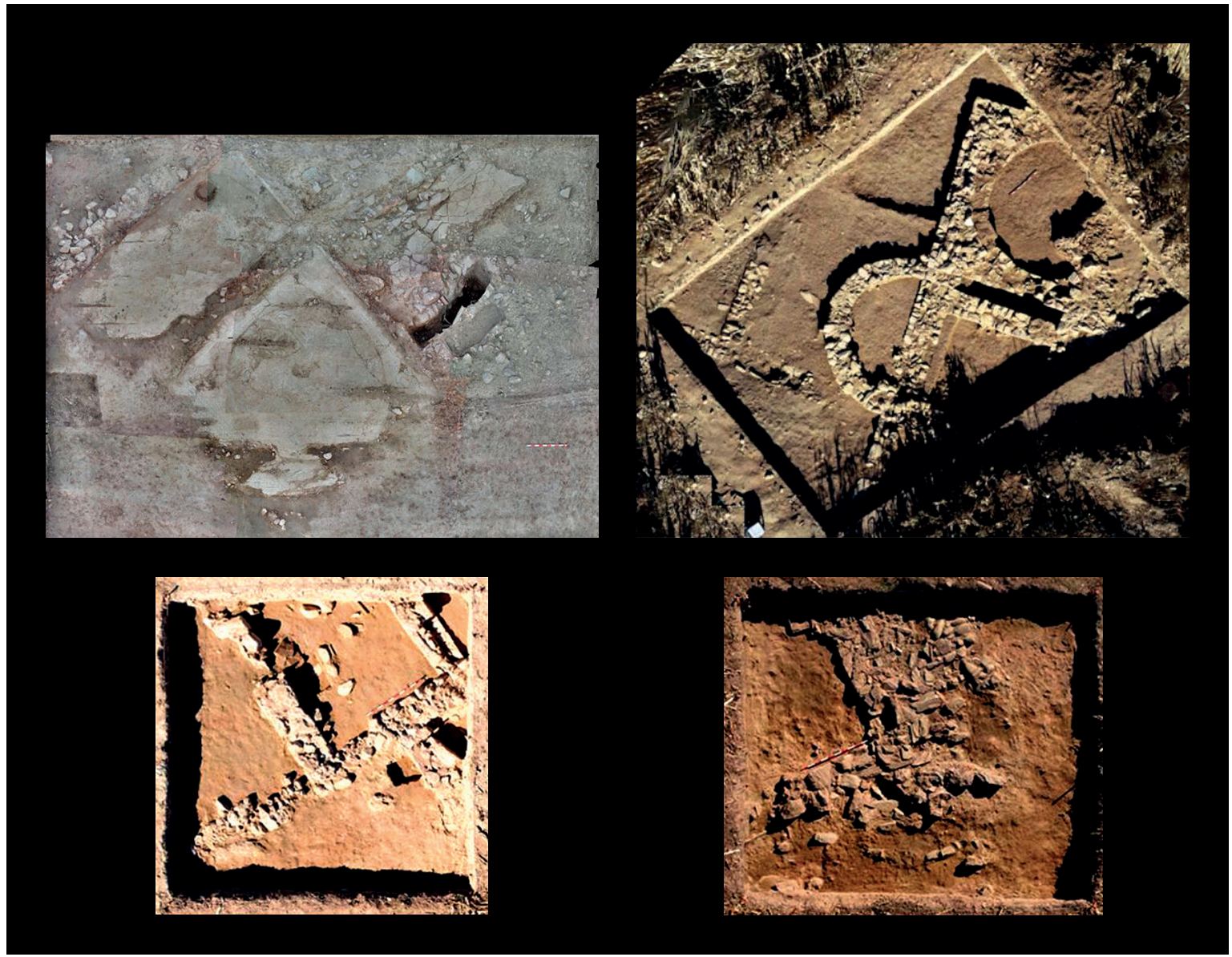

Figura 2

Estructuras documentadas en Los Villares durante tres campañas de excavación practicadas

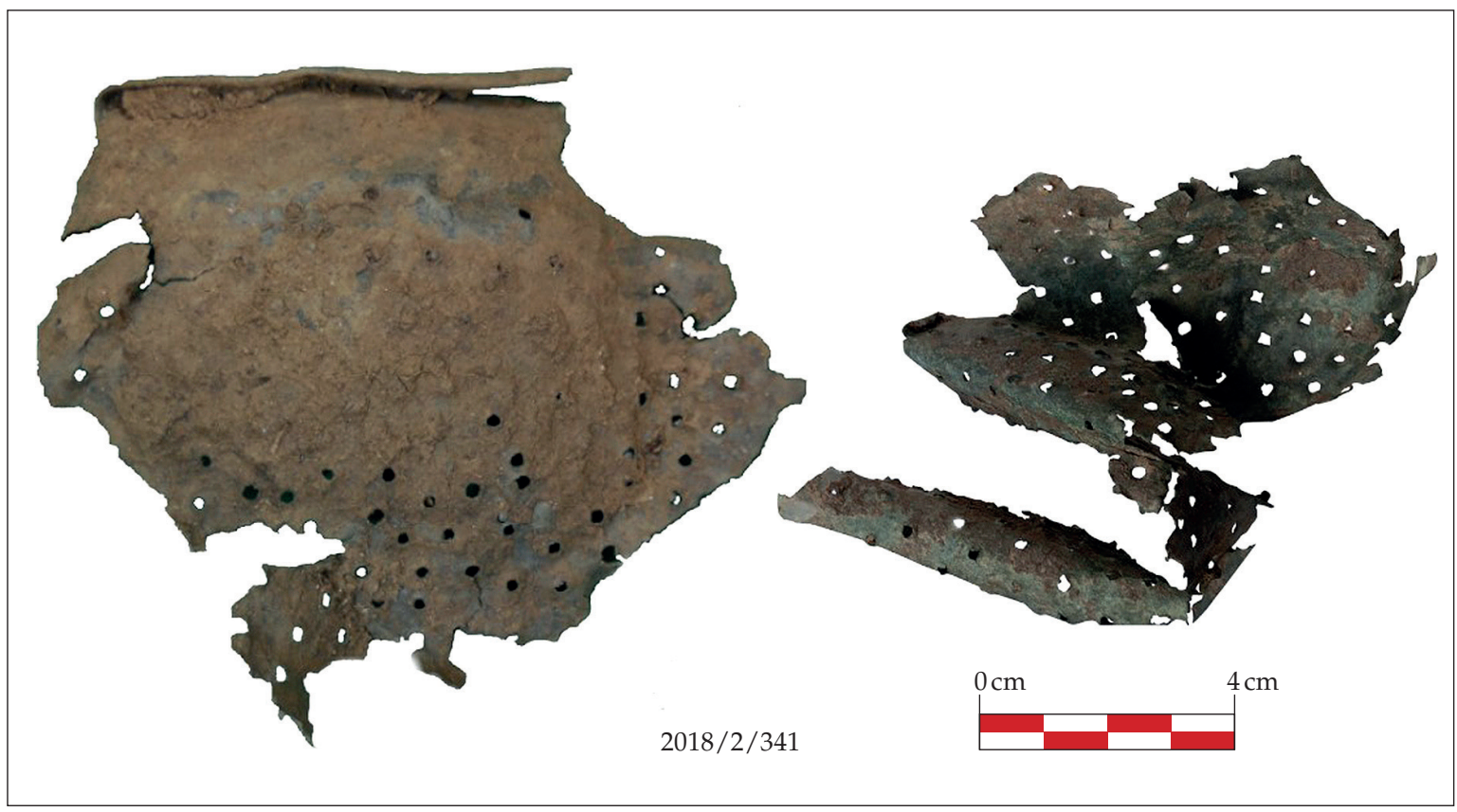

Figura 3

Partes de un colador hallados en el yacimiento de Los Villares (Fresno Alhándiga, Salamanca) 


\section{DESCRIPCIÓN DE LOS OBJETOS: UN LANX Y UN AEQUIPONDIUM}

Las piezas que se están analizando en este artículo fueron halladas en las campañas de excavación realizadas en el año 2017 y 2018. El platillo o lanx fue encontrado en superficie sin una estratigrafía concreta, pero el aequipondium fue hallado en excavación en un estrato de cronología tardorromana.

El lanx o plato de libra es un pequeño plato de bronce de forma circular, de $6.3 \mathrm{~cm}$ de diámetro. Su estado de conservación es aceptable, aunque posee unas pequeñas lagunas y pátina verdosa por la oxidación en ambas caras. Es un elemento que estaría vinculado a una balanza de la que no se han documentado restos hasta el momento. Fue localizado en superficie en el año 2017 junto a la cata realizada en el año 2015, por lo que las labores agrícolas del campo lo sacaron de su propia estratigrafía (n. inventario 2018/2/340). Otros objetos similares a este se han documentado en Segobriga (Cebrián y Hortelano, 2017: 2008) o en Regina Turdulorum (Pozo, 2002: 102, figura 100) (Figura 4).

El contrapeso o aequipondium fue hallado en la cata realizada en el año 2018. Se trata de una bola esférica de plomo recubierta de bronce con anillo de suspensión (0 inventario 2019/4/582). Los aequipondia solían realizarse en bronce y ser rellenos de

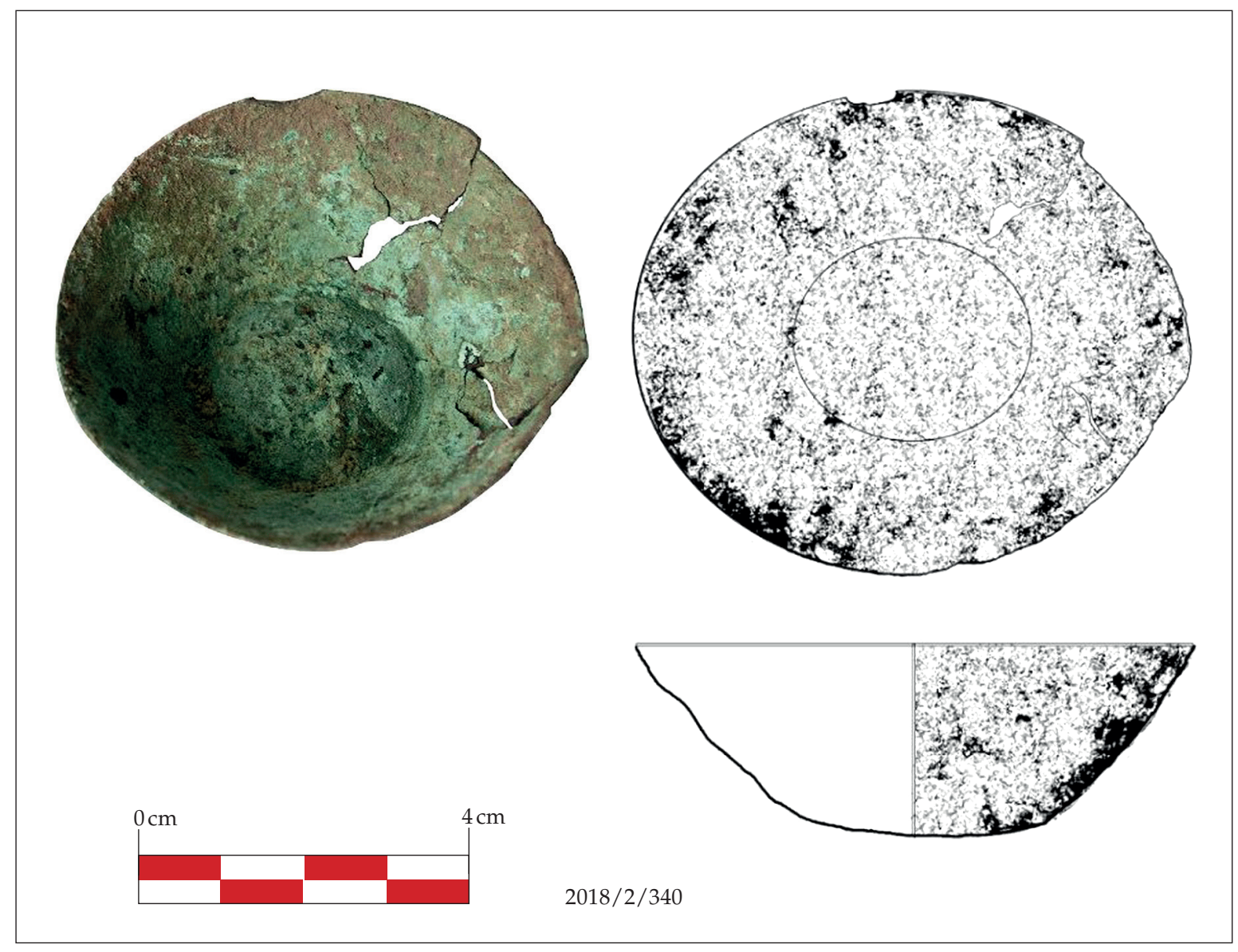

Figura 4

Fotografía y dibujo del Lanx hallado en superficie de Los Villares (Fresno Alhándiga, Salamanca) 
plomo para obtener pesos prefijados de la libra, la mayoría de ellos fueron utilizados para fines comerciales y/o domésticos, y suelen pesar una libra romana (de 327.45 o 322.56 gramos) (Rodríguez Martorell y Ruíz de Arbulo, 2016: 165-166). En este caso, su peso es algo mayor, $1 \mathrm{~kg}$, pero no es algo extraño en el mundo romano, donde se han hallado ejemplares de mayor tamaño como los recogidos en el trabajo de F. Chaves y R. Pliego (Chaves y Pliego 2007) o el aequipondium del puerto de Tarraco con $38 \mathrm{~kg}$ (Rodríguez Martorell y Ruíz de Arbulo, 2016:165). Presenta una morfología circular con el enganche realizado mediante una arandela en la parte superior. Parece apreciarse que el recubrimiento de bronce se realizó en dos partes que se juntaban en el centro. El recubrimiento estaba en malas condiciones de conservación por lo que no se pudo observar si conserva algún tipo de inscripción; sin embargo, parece intuirse unas líneas incisas que bordean el centro, todo ello podrá ser reconocido durante la restauración que se está llevando a cabo en el Museo Provincial de Salamanca. Formalmente sería del tipo B definido por R. Tyrrell, caracterizados por poseer aros de suspensión de hierro (aunque en el caso que se presenta sea de bronce) de formas diversas (Tyrrell, 2015) (Figura 5). Fue hallado en un estrato arcilloso amarillento (UE 4) en el que se recuperaron fragmentos cerámicos abundantes donde destacan la presencia de terra sigillata hispánica tardía y cerámica común, datado en época tardorromana.

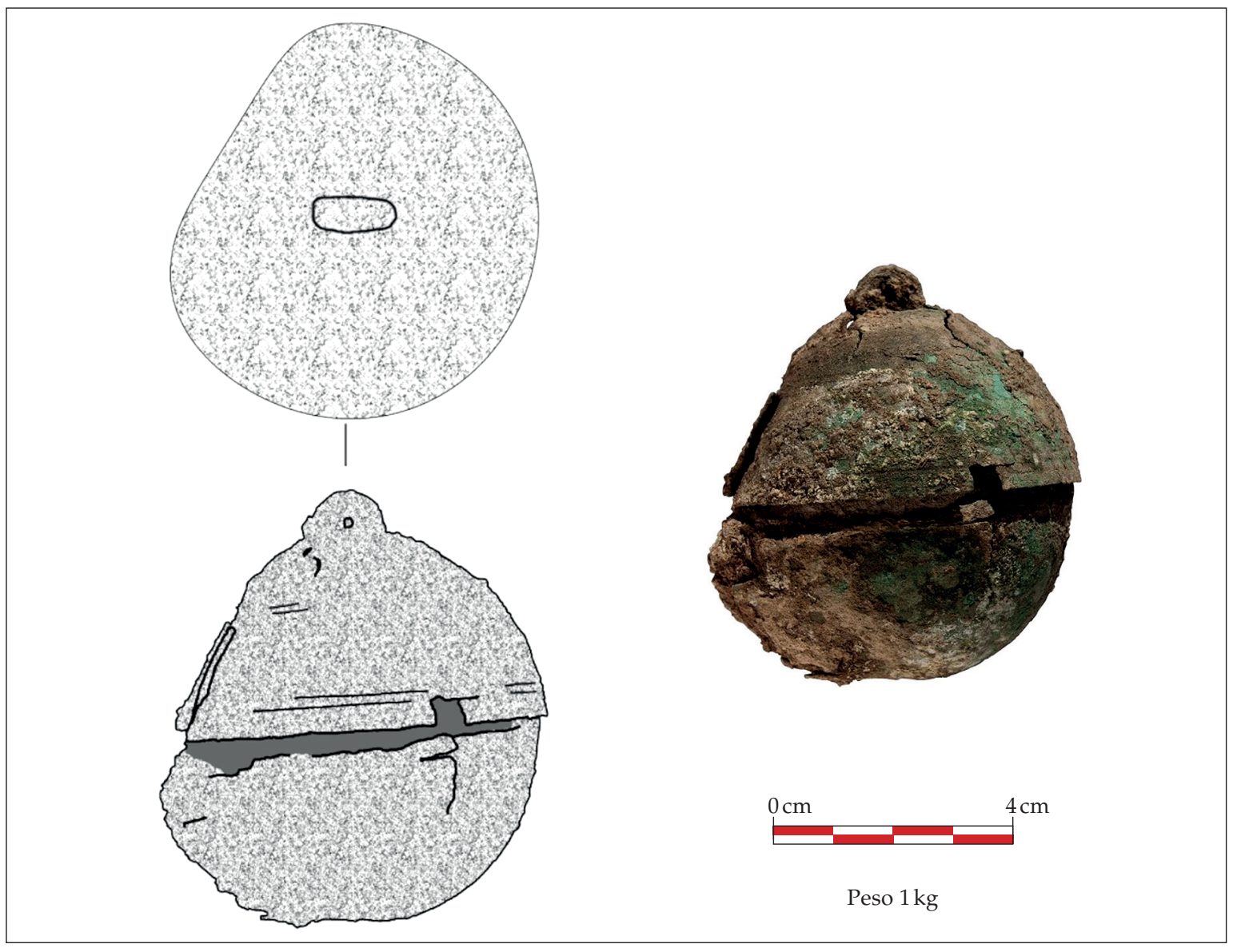

Figura 5

Dibujo y fotografía del Aequipondium de Los Villares (Fresno Alhándiga, Salamanca) 


\section{CONCLUSIONES}

Los dos objetos de bronce que se publican en este artículo pertenecen a piezas de una o varias balanzas o staterae halladas en un enclave con graves problemas de conservación. Sin embargo, aportan información sobre las propias transacciones que se podían estar llevando a cabo en el yacimiento del que proceden. En varios trabajos anteriores las autoras han defendido la importancia estratégica y la posible relación del enclave rural con la cercana vía de la Plata (Pérez y de Soto, 2017; de Soto y Pérez, 2019; Pérez et al., 2019); el hallazgo de elementos relacionados con la mesura sería un indicador más de la presencia constante de intercambios comerciales, ya no solo detectados por los contextos cerámicos con la llegada de producciones típicamente africanas (Pérez et al., 2019), sino también con sistemas de medida necesarios en la vida cotidiana de centros articuladores del territorio.

El material del que están hechos, bronce, no debe resultar extraño porque fue usado de forma abundante en la vida cotidiana de los romanos, siendo un elemento esencial tanto en la vida pública como en la privada. Su uso en la vida económica está atestiguado y cubría necesidades básicas como la vajilla doméstica o elementos decorativos (Rodríguez Oliva, 1990 93-97).

En resumen, durante la Antigüedad Tardía a pesar de las crisis y los problemas, las vías de comunicación debieron mantener su gran intensidad comercial como en el periodo precedente (Járrega, 2008: 62). La Vía de la Plata debió de continuar con un tránsito continuo de mercancías, productos manufacturados y personas, tal y como la arqueología poco a poco nos está dejando entrever mediante los hallazgos que se están produciendo en los últimos años en el yacimiento de Los Villares. 


\section{REFERENCIAS BIBLIOGRÁFICAS}

ARANEGUI, C. (1989): «Statera romana hallada en Valencia», Archivo de Prehistoria Levatina n. XIX, 263-264.

ARANEGUI, C. (1991): «Statera de bronce», C. Aranegui (Coord.): Saguntum y el mar, 107109 , Valencia.

CABALleRO, L. (1990) (comisario): Los Bronces Romanos en España. Catálogo de la exposición en el Palacio de Velázquez, Madrid.

CEBRIÁN FERNÁNDEZ, R.; HORTELANO UCEDA, I. (2017): «Librae, staterae y aequipondia de Segobriga. Instrumentos de pesar hallados en la ciudad y su entorno», Lvcentom n. XXXVI, 201-219. DOI: https://doi.org/10.14198/LVCENTVM2017.36.12

CHAVES, F. (1982): «Instrumentos de medida romanos hallados en Andalucía», Zephyros n. XXXIV-XXXV, 219-222. http://revistas.usal.es/index.php/0514-7336/article/view/1944

CHAVES, F. y PLIEGO, R. (2007): «Instrumentos de medida de pesos en la Hispania antigua», Sautuola n. ${ }^{\circ}$ XIII, 237-250.

CORTI, C. (2001): «Pesi e contrappesi», C. Corti y M. Giordani (Eds): Pondera. Pesi e mesure nell'Antichità, 191-212, Módena.

ERICE LACABE, Mª R. (1986): «Bronces romanos del Museo de Navarra», Trabajos de Arqueología Navarra n. 5, 195-235.

ERICE LACABE, M ${ }^{a}$ R. (2014): «Una pesa de balanza figurada procedente de Sofuentes, Zaragoza», A. Duplá, Mª V. Escribano, L. Sancho y Mª A. Villacampa (Eds.): Miscelánea de estudios en homenaje a Guillermo Fatás Cabeza, 257-263, Zaragoza.

ERICE LACABE, M ${ }^{a}$ R. (2015): «An «Aequipondium» from Sofuentes, Mid-Ebro valley, Spain», E. Deschler-Erb, Ph. Della Casa (eds.): New Research on An $\neg$ cient Bronzes. Acta of the XVIIIth International Congress on Ancient Bronzes, Zurich Studies in Archaeology $10,147-150$.

FERNÁNDEZ GÓMEZ, F. (1991): «Balanzas y romanas de bronce en los mercadillos de antigüedades de Sevilla», Trabajos de Prehistoria n. 48, 373-382.

https://doi.org/10.3989/tp.1991.v48.i0.535

JÁRREGA DOMINGUEZ, R. (2008): «Los estudios de cerámica romana en las zonas litorales de la Península Ibérica: un balance a inicios del siglo XXI», D. Bernal Casasola y A. Ribera (eds.): Cerámicas hispanorromanas. Un estado de la cuestión, 49-81, Cádiz.

PARES, Ch. 2013: «Weighing, commodification and money», H. Fokkens y A. Harding (eds.): The Oxford Handbook of the European Bronze Age, 508-523, Oxford.

PÉREZ DE DIOS, V.; DE SOTO GARCÍA, M. R. 2017: «Los Villares (Fresno Alhándiga, Salamanca): un complejo termal junto a la Vía de la Plata», Munibe n. 68, 237-249. https://doi: 10.21630/maa.2017.68.08

PÉREZ DE DIOS, V.; DE SOTO GARCÍA, M. R.; DE SOTO GARCÍA, I. S.; GARCÍA GIMÉNEZ, R. 2019: «La terra sigillata y la cerámica común africana en el yacimiento de Los Villares (Salamanca). Análisis del contexto cerámico y estudio arqueométrico», Actas del IV Congreso Internacional de la SECAH (Valencia, 2017), 491-502, Madrid. 
PÉREZ ZURITA, A. (2011): «Control y administración de pe $\neg$ sos y medidas en las ciudades del Imperio romano (Pars Occidentalis)», Gerión n. 29, 1, 123-148.

http:// dx.doi.org/10.5209/rev_geri.2011.v29.n1.39048.

PONTE, S. da (1979): «Balanças e pesos de Conimbriga», Conimbriga n. 18, 121-132.

POZO, S. F. (1994): «Pesas de balanza romanas de la Península Ibérica y las Islas Baleares», Akten der 10. Internationalen Tagungüber Antike Bronzen (Freiburg, 1988), 337-345, Stuttgart.

POZO, S. F. (2002): «Varia arqueológica de la provincia Baetica. Bronces romanos inéditos. Grandes bronces. Estatuillas. Mobiliario doméstico. Amuletos fálicos. Espejos. Balanzas. Contrapesos. Asas y apliques de sítulas. Atalaje de caballerías», Antiquitas n. ${ }^{14}$, 69-121.

RODRÍGUEZ MARTORELL, F. y RUIZ DE ARBULO, J. (2016): «Un aequipondium de peso excepcional y la balanza pública del puerto de Tarraco», Archivo Español de Arqueología n. 89, 163-180. DOI: http://dx.doi.org/10.3989/aespa.089.016.008

RODRÍGUEZ OLIVA, P. (1990): «Los bronces romanos de la Bética y la Lusitania», L. Caballero: Los Bronces Romanos en España. Catálogo de la exposición en el Palacio de Velázquez, 91-102, Madrid.

DE SOTO GARCÍA, M. R.; PÉREZ DE DIOS, V. 2019: «La combinación de la investigación con la protección del patrimonio arqueológico rural en la provincia de Salamanca: el caso de Los Villares (Fresno Alhándiga, Salamanca), Scientia Antiquitatis n. 3, 491-503. http://www.scientiaantiquitatis.uevora.pt/index.php/SA/article/view/241

TYRELL, R. (2015): «Lead weights», M. Atkinson y S. J. Preston (Eds.): Heybridge: A Late Iron Age and Roman Settle-ment, Excavations at Elms Farm 1993-5, Internet Archaeology, 40. DOI: http://dx.doi.org/10.11141/ia.40.1.tyrrell8 